\title{
Diagnostic Value of Contrast-Enhanced Ultrasound and Magnetic Resonance in the Diagnosis of Difficult to Determine Renal Tumor by Enhanced CT
}

xuhua Qiao

Kunming Medical University https://orcid.org/0000-0001-8350-5685

zhiyong Tan

Kunming Medical University Second Hospital

Ning Li

Kunming Medical University Second Hospital

Yigang Zuo

Kunming Medical University Second Hospital

Huake Wang ( $\nabla$ wanghuake@etyy.cn )

Kunming Medical University Second Hospital https://orcid.org/0000-0001-7996-7924

\section{Research Article}

Keywords: Contrast-enhanced CT is difficult to identify renal tumors, CEUS,MRI, Differential Diagnosis

Posted Date: May 28th, 2021

DOl: https://doi.org/10.21203/rs.3.rs-528185/v1

License: (9) This work is licensed under a Creative Commons Attribution 4.0 International License.

Read Full License 


\section{Abstract}

Objective

To analyze and compare the efficacy of contrast-enhanced ultrasound (CEUS) and magnetic resonance (MRI) in the diagnosis of difficult renal tumor with CT.

Methods

A total of 136 patients with renal space occupying who received CEUS and MRI in the Second Affiliated Hospital of Kunming Medical University from January 2013 to December 2020 after improved abdominal enhanced CT was difficult to distinguish between benign and malignant patients were retrospectively analyzed. The diagnostic results of the two examination methods were compared with the pathological examination results to analyze the diagnostic efficacy of the two methods.

Results

61 cases were benign and 75 cases were malignant. The sensitivity, specificity, accuracy, positive predictive value and negative predictive value of CEUS, MRI and combined examination for the differential diagnosis of benign and malignant renal tumors difficult to be determined by CT were (CEUS:94.42\%, 87.69\%, 91.18\%, 89.33\%, 93.44\%;MRI:81.63\%, 91.84\%, 85.29\%, 94.67\%,

73.77\%;MRI+CEUS:94.67\%, 93.44\%, 92.65\%, 94.67\%, 93.44\%). Comparison of the sensitivity of single and combined CEUS and MRI in the differential diagnosis of benign and malignant renal tumors that were difficult to be identified by $C T: \chi 2=11.34, P=0.001$, there was statistical significance; $\chi 2=0.86, P=0.35$, there was no statistical significance; $\chi 2=5.73, P=0.02$, between MRI and CEUS +CEUS.CEUS with single and combined MRI examination of CT qualitative differential diagnosis of benign and malignant renal tumors ROC curve shows that applied to the differential diagnosis of benign versus malignant tumor CT is qualitative the MRI + CEUS is better than that of CEUS, CEUS is better than that of MRI, the area under the curve comparison(accurate):MRI compar-ed with CEUC no statistical significance $(p=0.26)$, magnetic resonance imaging (MRI) and CEUS were compared with MRI + CEUS were statistically significant $(p=$ $0.02, p=0.01)$.

Conclusion

Both CEUS and MRI can be used to distinguish between benign and malignant renal tumors that are difficult to be characterized by CT. However, CEUS alone has more clinical application value in the diagnosis of benign and malignant renal tumors that are difficult to be characterized by CT considering economic effects, indications and side effects.

\section{Introduction}

Ultrasonogram,CT,MRI囚are useful radiological tools in diagnosis of renal tumor.Most renal tumors are found after ultrasound, CT, MRI and other imaging examinations. Due to the economical and practical 
type of ultrasound, ultrasound is currently the preferred examination method for primary screening of renal tumors $[1,2]$.Identifying the benign and malignant renal tumors from imaging has guiding significance for the selection of treatment methods. Identifying the benign and malignant renal tumors as early as possible can help patients choose the optimal treatment plan. The diagnosis of benign and malignant renal tumor gold standard is pathology examination, but the pathological examination for have a check, not the first choice for the benign and malignant tumor as a clear plan, benign and malignant tumor imaging examination is the identification of the main basis, but not yet studies have shown that any kind of imaging examination in benign and malignant tumor in the identification accuracy can reach $100 \% .[3-5]$.

Different imaging examinations have different accuracy in the differential diagnosis of benign and malignant renal tumors. CT, as a commonly used means of clinical diagnosis for the differentiation of benign and malignant renal tumors and stages, has obvious advantages compared with contrast enhanced ultrasound and MRI in renal tumors with diameter less than $3 \mathrm{~cm}$ and cystic renal tumors and other complex renal space occupying [6, 7].Current studies suggest that the combination of B-mode ultrasound, CT and MRI for some atypical renal space-occupying lesions such as small diameter and complex lesions can improve the accuracy of differentiating benign and malignant renal tumors [8, 9].A number of meta-analyses suggest that CEUS has a high accuracy in differentiating benign and malignant renal cysts with small renal mass and complex renal cysts, which is higher than or equal to CT and MRI. Compared with CT, CEUS can reduce the use of nephrotoxic contrast agents, and can reduce the economic burden of the medical care system compared with MRI.But all the studies suggest that more research is needed [10-12].

CEUS has been widely used in the qualitative diagnosis of tumors. At present, existing studies have shown that CEUS is superior to CT. Therefore, it is suggested to promote the application of CT combined with CEUS in the qualitative diagnosis of complex renal tumors $[13,14]$.MRI is also widely used in the qualitative diagnosis and staging of tumors, but there are currently no studies to compare the accuracy and clinical application value of CEUS and MRI in complex renal tumors. The application of CEUS to the qualitative diagnosis of renal tumors is gradually being promoted in the clinic. Combined with other imaging examinations, the accuracy of the qualitative diagnosis of tumors can be improved. This study reviewed and analyzed the cases in our hospital and compared CEUS and MRI on enhanced CT for difficult to characterize renal tumors. The clinical value of the differential diagnosis of malignancy is to compare the pros and cons of the two tests.

\section{Clinical Remarks}

A retrospective analysis was performed in the Second Affiliated Hospital of Kunming Medical University from January 2013 to December 2020. A total of 136 cases of renal occupying cases, which were difficult to distinguish between benign and malignant renal masses, were performed by abdominal enhanced CT outside the hospital. After admission, these patients have undergone renal magnetic resonance imaging (MRI) and contrast-enhanced ultrasound (CEUS). Among them, 67 were males and 69 were females; they 
were 27 to 80 years old, with an average age of 57 years. After admission, 7 patients underwent flexible ureteroscopy, and 129 patients underwent surgical treatment and sent specimens for medical examination. All patients had no contraindications to MRI and CEUS examinations, and they voluntarily signed an informed consent form before the examination.

\section{Diagnostic Methods}

2.1 CEUS uses Mylab Twice color Doppler ultrasound system and GE Logic 9 color Doppler ultrasound system. The probe frequency is $2-4.5 \mathrm{MHz}$. First, use the conventional ultrasound mode to determine the best examination surface for renal tumors, switch the examination mode to CEUS mode, use sonovi contrast agent, dilute and mix with $5 \mathrm{~mL}$ of saline for injection, and extract $2 \mathrm{ml}$ of the contrast agent through the anterior cubital vein for rapid bolus injection, Inject $5 \mathrm{~m}$ normal saline into the tube, continuously observe and enhance CT imaging of difficult-to-characterized renal tumor lesions and surrounding tissues for 3-5 minutes. While injecting the contrast agent, start the built-in timer of the instrument and store dynamic images. After the contrast, the ultrasound diagnosis physician Analyze the image to get a color Doppler ultrasound report.

2.2 MRI imaging uses GE1.5T superconducting scanner to routinely perform MRI plain scan and dynamic enhanced scan. All patients had undergone multi-parameter MRI examination before the biopsy. Before the examination, the patients were instructed to abstain from drinking for 4 hours, and the patients were instructed to apnea training. All patients were given T1WI/T2WI plain scans of the transverse and coronal planes. Quickly inject $15 \mathrm{ml}$ of contrast agent (Gd-DTPA) from the elbow vein at a speed of $2 \mathrm{ml} / \mathrm{s}$, and perform dynamic enhanced scanning, divided into renal cortex, renal medulla, excretion and renal pelvic phase. Delayed scanning of renal pelvic phase $4 \llbracket 6 \mathrm{~min}$.

2.3 Histopathology After surgical resection of the renal mass, the patient will be sent for pathological biopsy immediately, and the pathology department will perform the corresponding treatment and then cut the section and perform immunohistochemical staining on the specimen.

2.4 Statistical Methods The sensitivity, specificity, and accuracy of CEUS, MRI and the combined examination method for enhancing the sensitivity, specificity and accuracy of the differential diagnosis of benign and malignant renal tumors with difficult to characterize on CT are expressed as a rate (\%). SPSS 25.0 statistical software was used for data analysis, CEUS and MRI and combined examination methods were used to compare the sensitivity of enhanced CT in the differential diagnosis of benign and malignant renal tumors. The chi-square test was compared. $\mathrm{P}<0.05$ was considered statistically significant; for CEUS and Logistic regression analysis of MRI results was performed to obtain the predicted probability of combined diagnosis, and then the predicted probability, CEUS and MRI results were used to create the ROC curve for the differential diagnosis of benign and malignant renal tumors with enhanced CT using CEUS and MRI and combined methods. Use Medcalc19.0.4 statistical software to compare the area under the ROC curve, perform $\mathrm{Z}$ test, and $\mathrm{P}<0.05$ is considered statistically significant. 


\section{Results}

3.1 Among the 136 patients with renal tumors that were difficult to characterize with enhanced $\mathrm{CT}, 75$ were malignant tumors, accounting for $55.15 \%$ of all cases, and 61 were benign tumors, accounting for $44.85 \%$ of all cases. Among them, according to the type of pathology, the number of specific pathological types and CEUS and MRI alone and in combination for the diagnosis of benign and malignant types of kidney tumors with different pathological types that are difficult to characterize with enhanced CT are shown in Table 1.

3.2 Statistical analysis of the sensitivity of CEUS and MRI alone or in combination in the differential diagnosis of benign and malignant renal tumors with enhanced CT

The sensitivity, specificity and accuracy of CEUS and MRI alone and in combination for the diagnosis of benign and malignant lesions of difficult-to-characterized kidney tumors with enhanced CT are shown in Table 2 (Table 2). , Positive predictive value, negative predictive value (Table 3). CEUS and MRI alone and in combination with enhanced CT in the differential diagnosis of benign and malignant renal tumors that are difficult to characterize (Table 4). The sensitivity comparison of CEUS and MRI alone and in combination in the differential diagnosis of benign and malignant renal neoplasms with enhanced CT: $\chi 2=6.21, p=0.04$. The comparison of sensitivity between CEUS and MRI alone in the differential diagnosis of benign and malignant renal tumors with difficult-to-enhance enhanced CT: $\chi 2=11.34, p=0.001$. Comparison of sensitivity between CEUS and MRI+CEUS in the differential diagnosis of benign and malignant renal tumors with enhanced $C T: \chi 2=0.86, p=0.35$. Comparison of sensitivity between MRI and MRI+CEUS in the differential diagnosis of benign and malignant renal neoplasms with enhanced CT: $\chi 2=5.73, p=0.02$.

\subsection{The statistical analysis of the accuracy of CEUS and MRI alone or in combination in the differential diagnosis of benign and malignant renal tumors with enhanced CT}

The ROC curve of CEUS and MRI alone and in combination for the differential diagnosis of benign and malignant renal tumors with enhanced CT is shown (Figure 1). The area under the ROC curve of CEUS, MRI, and CEUS combined with MRI are $91.4 \%, 84.2 \%$, and $94.1 \%$, respectively. The comparison of the area under the ROC curve between MRI and CEUC was not statistically significant $(p=0.26)$, that is, CEUS and MRI had no difference in the accuracy of the differential diagnosis of benign and malignant renal tumors with difficult to characterize enhanced CT. The area under the ROC curve of MRI and CEUS were statistically significant compared with the area under the ROC curve of MRI combined with CEUS $(p=0.02$, $p=0.01$ ), that is, MRI combined with CEUS can distinguish benign and malignant renal tumors that are difficult to characterize with enhanced CT The accuracy of diagnosis is higher than that of MRI and CEUS alone.

\section{Discussion}


The main method of screening for kidney tumors in healthy people is ultrasound, which is also the most commonly used examination method for the diagnosis of kidney tumors. During ultrasound examination, most of the sonograms of renal malignant tumors are hypoechoic or isoechoic, and a small number of them are hyperechoic; the presence of anechoic areas in the tumor and hypoechoic halo around the tumor is also considered to be an indication for judging malignancy , But some renal malignant tumors do not have these characteristics, and it is necessary to use CT or MRI for differential diagnosis [15].The sensitivity and specificity of ultrasound in the diagnosis of renal malignant tumors are closely related to the size of renal tumors. The sensitivity of ultrasound to detect renal malignant tumors with a maximum tumor diameter of $<5 \mathrm{~mm}$ is almost zero, but CT can reach more than $40 \%$, which is the largest for tumors. For renal tumors larger than $30 \mathrm{~mm}$ in diameter, the sensitivity of ultrasound and CT detection can reach $100 \%[16,17]$.

In recent years, the research of ultrasound contrast agents has made progress. Intravenous injection of ultrasound contrast agents can improve the echo of blood flow and enhance the Doppler signal. It can well show the branches of blood vessels in the kidney, the renal tissue and the peripheral or internal small blood vessels of the tumor. The perfusion condition improves the diagnostic accuracy rate of renal masses, especially in the diagnosis of cystic renal cancer or cyst inner wall nodules or cyst malignant transformation. It can significantly improve the low blood flow display rate of ordinary color Doppler ultrasound, thereby confirming the diagnosis, and Increased the coincidence rate of ultrasound and pathological diagnosis [18].After injection of ultrasound contrast agent, the blood flow display in benign and malignant tumors is correspondingly enhanced, but the enhancement degree and duration are significantly different. The enhancement degree of malignant tumor blood flow imaging is significantly higher than that of benign tumors (except renal hemangioma). The clearance of the agent is also faster than that of benign tumors, and the benign and malignant tumors can be judged based on these characteristics [19].

The identification and detection rate of CEUS for small tumors is higher than that of enhanced CT, and the scope of application, cost-effectiveness, inspection time and radiation of CEUS are all lower than that of enhanced CT. [20, 21].The Meta-analysis of the value of CEUS and enhanced CT in the differential diagnosis of renal cystic space occupying benign and malignant is believed to be better than CEUS in the differential diagnosis of renal cystic space occupying benign and malignant based on its clinical application value [22].In recent years, clinical studies have concluded that the accuracy and sensitivity of CEUS in the differential diagnosis of benign and malignant renal tumors are significantly better than that of $C T$, but the advantages of CEUS and CT in clinical staging and three-dimensional reconstruction need to be explored [23].CEUS and MRI have a higher compliance rate in pathological diagnosis of complex cystic than CT. Both CEUS and MRI can be used to evaluate complex cystic kidney masses. CEUS is more sensitive than MRI but has lower specificity [24, 25].Marschner et al[26] Studies have shown that CEUS, MRI and CT are equally accurate in distinguishing benign and malignant kidney tumors, but CEUS can be applied to patients with contraindications in MRI and CT examinations. 
This study retrospectively analyzed the clinical value of CEUS and MRI alone and combined with enhanced CT in the diagnosis of benign and malignant renal tumors. The results of the study showed that CEUS and MRI alone and combined with enhanced CT were effective in the differential diagnosis of benign and malignant renal tumors. It is statistically significant $(p<0.05)$, that is, the diagnostic sensitivity of the three inspection programs is different. The sensitivity of CEUS and MRI alone in the differential diagnosis of benign and malignant renal tumors with enhanced CT is statistically significant $(p<0.05)$, that is, CEUS scheme is more sensitive than MRI in the differential diagnosis of benign and malignant renal tumors with enhanced CT. It can be seen from Table 1 that in this study, CEUS and MRI have almost the same malignant detection rates of renal cystic and solid renal masses. The current study also believes that CEUS and MRI are in the diagnosis of complex renal cystic. MRI is more sensitive than MRI in distinguishing benign and malignant complicated renal cystic diseases.[27, 28].CEUS and MRI+CEUS have no statistical significance in the differential diagnosis of benign and malignant renal tumors with enhanced CT ( $p>0.05)$, that is, there is no difference in the diagnostic sensitivity of the two inspection schemes. MRI and MRI+CEUS have no statistical significance in the differential diagnosis of renal tumors with enhanced CT. The sensitivity of the differential diagnosis of benign and malignant is statistically significant $(p<0.05)$, that is, the sensitivity of MRI+CEUS in enhancing the diagnosis of benign and malignant renal tumors with difficult to determine CT is better than that of MRI. Although MRI+CEUS improves the sensitivity, it is in actual clinical practice. The application value of MRI is not high, the price of MRI is expensive, the inspection is time-consuming, and the sensitivity is not much different from the sensitivity of CEUS alone. In this study, the diagnosis results of CEUS alone were consistent with pathology in 124 cases. MRI+ There are 128 cases of CEUS[29]. The ROC curve analysis of CEUS and MRI alone or in combination in the differential diagnosis of benign and malignant renal tumors with enhanced CT. The value of CEUS and MRI in the differential diagnosis of benign and malignant renal tumors with enhanced CT is that CEUS+MRI is better than CEUS. In terms of accuracy comparison between CEUS and $\mathrm{MRI}$, the combination of the two examinations can improve the accuracy of the diagnosis of benign and malignant renal tumors that are difficult to characterize by enhanced $\mathrm{CT}$, but there is no significant difference in the accuracy of separate examinations, so the combined examination can indeed improve the correct diagnosis of tumors. However, the clinical application value of this combination of multiple imaging examinations needs to be further explored [30].

In summary, CEUS is better than MRI in the differential diagnosis of benign and malignant renal tumors with enhanced CT, but its specificity is lower than that of MRI. Both CEUS and MRI can be used to differentiate between benign and malignant renal tumors with enhanced CT. The combined diagnosis of these examinations can improve the efficiency of enhancing the differential diagnosis of benign and malignant renal tumors with difficult CT. However, from the consideration of economic effects, indications, and side effects, CEUS alone has more clinical application value in the differentiation of benign and malignant renal tumors with difficult CT. Combined with this study, the best plan for the diagnosis of renal tumors with difficult-to-characterized enhanced CT is ultrasound and contrastenhanced ultrasound, and then MRI is selected according to the needs of clinical diagnosis and treatment. 


\section{Declarations}

Acknowledgements

There is no special explanation for the content of acknowledgement.

Authors' contributions

Xuhua Qiao: data acquisition, literature research, and manuscript writing.Zhiyong Tan®Yigang Zuo,Ning $\mathrm{Li}$ : data acquisition and review, Huake Wang: study design and theoretical support. Zhiyong Tan: design of the research program, review and revise of manuscript. All the authors agreed on the content of the final manuscript.

\section{References}

1. Will H: [Ultrasound diagnosis of the kidney].Die Medizinische Welt 1981, 32(22):847-852.

2. Correas JM, Anglicheau D, Joly D, Gennisson JL, Tanter M, Hélénon O: Ultrasound-based imaging methods of the kidney-recent developments. KIDNEY INT 2016, 90(6):1199-1210.

3. Motzer RJ, Jonasch E, Michaelson MD, Nandagopal L, Gore JL, George S, Alva A, Haas N, Harrison MR, Plimack ER et al: NCCN Guidelines Insights: Kidney Cancer, Version 2.2020. Journal of the National Comprehensive Cancer Network: JNCCN2019, 17(11):1278-1285.

4. Hommerding O, Allory Y, Argani P, Bismar TA, Bubendorf L, Canete-Portillo S, Chaux A, Chen YB, Cheng L, Cubilla AL et al: [Molecular pathology of urogenital tumors : Recommendations from the 2019 International Society of Urological Pathology (ISUP) Consensus Conference].Der Pathologe 2021.

5. Campbell S, Uzzo RG, Allaf ME, Bass EB, Cadeddu JA, Chang A, Clark PE, Davis BJ, Derweesh IH, Giambarresi $L$ et al: Renal Mass and Localized Renal Cancer: AUA Guideline. The Journal of urology 2017, 198(3):520-529.

6. Kang SK, Kim D, Chandarana $\mathrm{H}$ : Contemporary imaging of the renal mass. CURR UROL REP 2011, 12(1):11-17.

7. Krishna S, Leckie A, Kielar A, Hartman R, Khandelwal A: Imaging of Renal Cancer.Seminars in ultrasound, CT, and MR 2020, 41(2):152-169.

8. Ziegelmüller BK, Spek A, Szabados B, Casuscelli J, Clevert DA, Staehler M: [Epidemiology and diagnostic assessment of small renal masses].Der Urologe. Ausg. A 2018, 57(3):274-279.

9. Graumann O, Osther SS, Karstoft J, Hørlyck A, Osther PJ: Bosniak classification system: a prospective comparison of CT, contrastenhanced US, and MR for categorizing complex renal cystic masses.Acta radiologica (Stockholm, Sweden : 1987) 2016, 57(11):1409-1417.

10. Wu JF, Wu C, Wang YL, Wang ZP: Diagnostic accuracy of contrastenhanced ultrasound in the detection of small renal masses: A protocol of systematic review and meta-analysis.MEDICINE 2020, 99(29):e21262. 
11. Shen L, Li Y, Li N, Zhao Y, Zhou Q, Li Z: Clinical utility of contrastenhanced ultrasonography in the diagnosis of benign and malignant small renal masses among Asian population.CANCER MED-US 2019, 8(18):7532-7541.

12. Furrer MA, Spycher S, Büttiker SM, Gross T, Bosshard P, Thalmann GN, Schneider MP, Roth B: Comparison of the Diagnostic Performance of Contrastenhanced Ultrasound with That of Contrastenhanced Computed Tomography and Contrast-enhanced Magnetic Resonance Imaging in the Evaluation of Renal Masses: A Systematic Review and Meta-analysis.European urology oncology 2020, 3(4):464-473.

13. Wei SP, Xu CL, Zhang Q, Zhang QR, Zhao YE, Huang PF, Xie YD, Zhou CS, Tian FL, Yang B: Contrastenhanced ultrasound for differentiating benign from malignant solid small renal masses: comparison with contrast-enhanced CT.Abdominal radiology (New York) 2017, 42(8):2135-2145.

14. Nicolau C, Buñesch L, Paño B, Salvador R, Ribal MJ, Mallofré $C$, Sebastià C: Prospective evaluation of $\mathrm{CT}$ indeterminate renal masses using US and contrast-enhanced ultrasound.ABDOM IMAGING 2015, 40(3):542-551.

15. Porena M, Del Zingaro M, Fornetti P, Salomone U, Rosi P: [Ultrasonography and Doppler color ultrasonography in kidney neoplasms: the demands of the clinician].Archivio italiano di urologia, andrologia : organo ufficiale [di] Societa italiana di ecografia urologica e nefrologica 2000, 72(4):135137.

16. Dalla-Palma L, Pozzi-Mucelli R: Problematic renal masses in ultrasonography and computed tomography. CLIN IMAG 1990, 14(2):83-98.

17. Kim JH, Li S, Khandwala Y, Del Giudice F, Chung KJ, Park HK, Chung BI: National trends of preoperative imaging modalities before partial nephrectomy for renal masses in the U.S. from 20072015. Canadian Urological Association journal = Journal de l'Association des urologues du Canada 2019, 13(3):E89-E94.

18. Geyer T, Schwarze V, Marschner C, Schnitzer ML, Froelich MF, Rübenthaler J, Clevert DA: Diagnostic Performance of Contrast-Enhanced Ultrasound (CEUS) in the Evaluation of Solid Renal Masses.Medicina (Kaunas, Lithuania) 2020, 56(11).

19. Siracusano S, Bertolotto M, Ciciliato S, Valentino M, Liguori G, Visalli F: The current role of contrastenhanced ultrasound (CEUS) imaging in the evaluation of renal pathology.WORLD J UROL 2011, 29(5):633-638.

20. Bertelli E, Palombella A, Sessa F, Baldi I, Morelli N, Verna S, Greco I, Morselli S, Pili A, Sebastianelli A et al: Contrast-enhanced ultrasound (CEUS) imaging for active surveillance of small renal masses. WORLD J UROL 2021.

21. Atri M, Tabatabaeifar L, Jang HJ, Finelli A, Moshonov H, Jewett M: Accuracy of Contrastenhanced US for Differentiating Benign from Malignant Solid Small Renal Masses.RADIOLOGY 2015, 276(3):900-908.

22. Lan D, Qu HC, Li N, Zhu XW, Liu YL, Liu CL: The Value of Contrast-Enhanced Ultrasonography and Contrast-Enhanced CT in the Diagnosis of Malignant Renal Cystic Lesions: A Meta-Analysis. PLOS 
ONE 2016, 11(5):e155857.

23. Thaiss WM, Bedke J, Kruck S, Spira D, Stenzl A, Nikolaou K, Horger M, Kaufmann S: Can contrastenhanced ultrasound and acoustic radiation force impulse imaging characterize CT-indeterminate renal masses? A prospective evaluation with histological confirmation. WORLD J UROL 2019, 37(7):1339-1346.

24. Rübenthaler J, Negrão De Figueiredo G, Mueller-Peltzer K, Clevert DA: Evaluation of renal lesions using contrast-enhanced ultrasound (CEUS); a 10-year retrospective European single-centre analysis.EUR RADIOL 2018, 28(11):4542-4549.

25. Rübenthaler J, Čečatka S, Froelich MF, Stechele M, Marschner C, Sabel BO, Bogner F, Schnitzer ML, Overhoff D, Große Hokamp N et al: Contrast-Enhanced Ultrasound (CEUS) for Follow-Up of Bosniak 2F Complex Renal Cystic Lesions-A 12-Year Retrospective Study in a Specialized European Center.CANCERS 2020, 12(8).

26. Marschner CA, Ruebenthaler J, Schwarze V, Negrão De Figueiredo G, Zhang L, Clevert DA: Comparison of computed tomography (CT), magnetic resonance imaging (MRI) and contrastenhanced ultrasound (CEUS) in the evaluation of unclear renal lesions.RoFo : Fortschritte auf dem Gebiete der Rontgenstrahlen und der Nuklearmedizin 2020, 192(11):1053-1059.

27. Rübenthaler J, Paprottka KJ, Marcon J, Reiser M, Clevert DA: MRI and contrast enhanced ultrasound (CEUS) image fusion of renal lesions.CLIN HEMORHEOL MICRO 2016, 64(3):457-466.

28. Chen Y, Wu N, Xue T, Hao Y, Dai J: Comparison of contrastenhanced sonography with MRI in the diagnosis of complex cystic renal masses.Journal of clinical ultrasound : JCU 2015, 43(4):203-209.

29. Eisner $\mathrm{BH}$, Kurtz MP, Harisinghani MG: Evolving role of magnetic resonance imaging in renal cancer imaging.J ENDOUROL 2010, 24(5):707-711.

30. Schwarze V, Rübenthaler J, Marschner C, Fabritius MP, Rueckel J, Fink N, Puhr-Westerheide D, Gresser $\mathrm{E}$, Froelich MF, Schnitzer ML et al: Advanced Fusion Imaging and Contrast-Enhanced Imaging (CT/MRI-CEUS) in Oncology.CANCERS 2020, 12(10).

\section{Tables}

Due to technical limitations, table 1, 2, 3 and 4 is only available as a download in the Supplemental Files section.

\section{Figures}




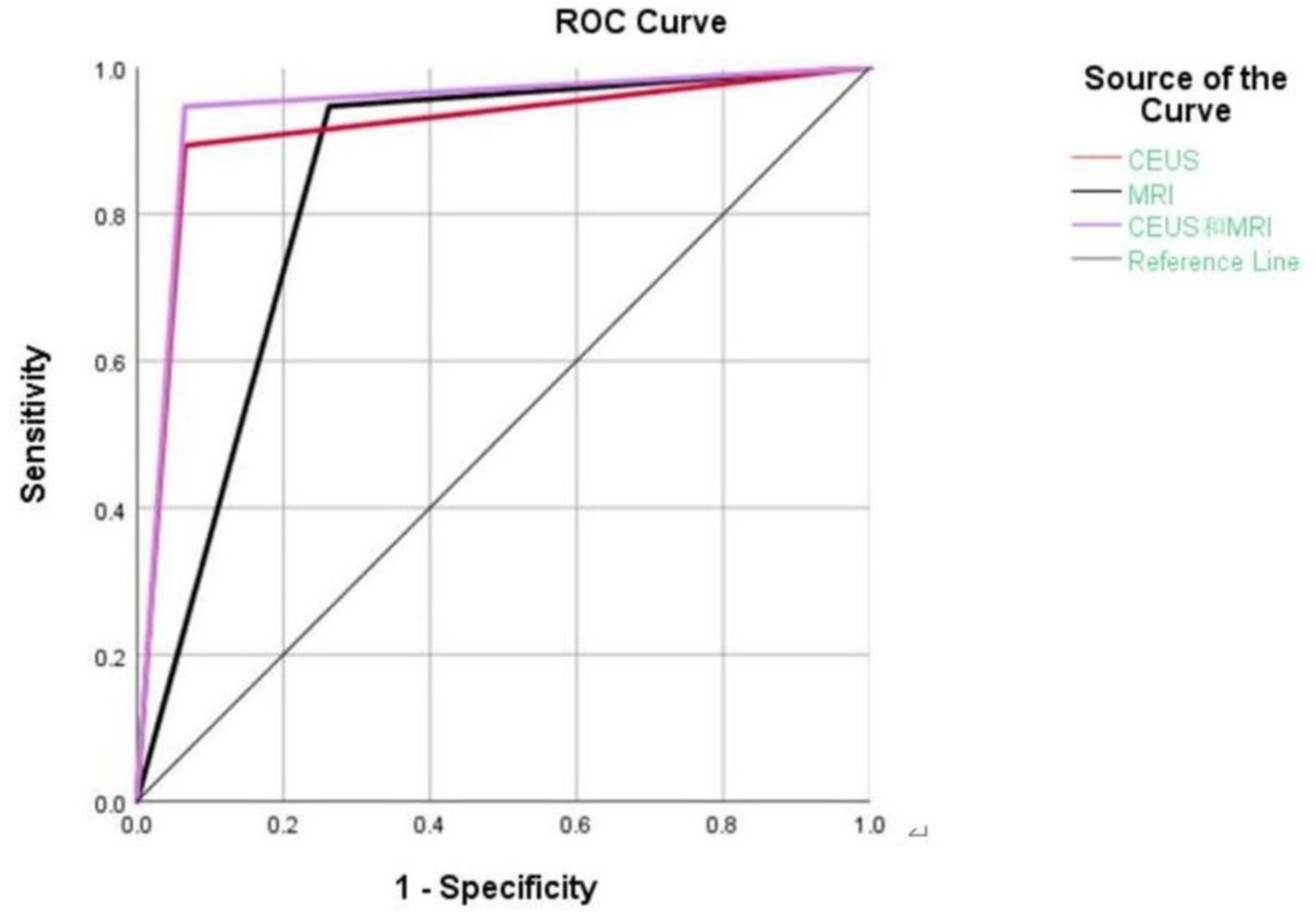

Figure 1

ROC curves of the differential diagnosis of benign and malignant renal tumors difficult to be determined by enhanced CT alone or in combination with CEUS and MRI

\section{Supplementary Files}

This is a list of supplementary files associated with this preprint. Click to download.

- Table.pdf 